\title{
Continuous Fuel Level Sensor Based on Spiral Side-Emitting Optical Fiber
}

\author{
Chengrui Zhao, Lin Ye, Xun Yu, and Junfeng Ge \\ Department of Control Science and Engineering, Huazhong University of Science and Technology, \\ 1037 Luoyu Road, Hubei, Wuhan 430074, China
}

Correspondence should be addressed to Junfeng Ge, gejf@mail.hust.edu.cn

Received 1 July 2012; Revised 15 October 2012; Accepted 24 October 2012

Academic Editor: Derong Liu

Copyright (c) 2012 Chengrui Zhao et al. This is an open access article distributed under the Creative Commons Attribution License, which permits unrestricted use, distribution, and reproduction in any medium, provided the original work is properly cited.

A continuous fuel level sensor using a side-emitting optical fiber is introduced in this paper. This sensor operates on the modulation of the light intensity in fiber, which is caused by the cladding's acceptance angle change when it is immersed in fuel. The fiber is bent as a spiral shape to increase the sensor's sensitivity by increasing the attenuation coefficient and fiber's submerged length compared to liquid level. The attenuation coefficients of fiber with different bent radiuses in the air and water are acquired through experiments. The fiber is designed as a spiral shape with a steadily changing slope, and its response to water level is simulated. The experimental results taken in water and aviation kerosene demonstrate a performance of $0.9 \mathrm{~m}$ range and $10 \mathrm{~mm}$ resolution.

\section{Introduction}

Fuel level measurement is a great challenge in aircraft fuel systems [1]. The most frequently used level sensor for aircraft is the capacitive level sensor for its good sensitivity and maintainability. But when the plane takes off from an airport with hot and wet environment, the moisture in the fuel tank will be congealed and mixed in the fuel, then error and short circuit will happen to the capacitive sensor. For this reason, ultrasonic level sensor was used to replace the capacitive sensor in the planes like B777, F22, and so forth. Now the fuel level sensors on the newest aircrafts like B787, A380, and F35 are changed back to the capacitive type, for the ultrasonic level sensor's bad performance in reliability and maintainability during the flight.

Optical fiber liquid level sensors (OFLLS) have been reported to be safe and reliable and have many advantages for aircraft fuel measurement [2]. Above all, the moisture or water mixed in the fuel will have little influence to the OFLLS because the optical fiber is nonconducting. Many OFLLS have been developed, such as float type [3], pressure type [4], optical radar type [5], and TIR type [6]. Side-leaking OFLLSs can make the conducting light leaking from the side of the fiber and have different attenuation coefficients in air and liquid [7]. Compared to other kinds of OFLLS, the main advantages of the side-leaking OFLLSs are immune to air pressure and insensitive to liquid sloshing and have no moving parts. There are many implementations for optical fiber side leakage: reducing the thickness of fiber cladding [8]; removing several zones of the cladding [9]; using fluorescent impurity-doped fiber [10]; side-polishing the cladding and a portion of core on a curved fiber [11]; using an etched FBG [12], or using a tilted FBG [13]. Until now, these sensors have not been commercialized due to low sensitivity $[9,11]$, limited range $[12,13]$, expensive cost $[8]$, and complicated manufacturing $[8,12,13]$.

Side-emitting optical fiber (SEOF) is a kind of polymer optical fiber of which the conducting light can leak from the side of fiber, and it is usually used for illumination and framing of buildings [14]. Compared to the side-leaking methods mentioned above, the light attenuation of SEOF is uniform, and its manufacturing cost is very low. There are many kinds of SEOF, and the one produced by cladding material crystallization is chosen for liquid level sensing, because it has a larger side emitting intensity compared with other SEOFs [15]. In this kind of fiber, there are many tiny crystals in the cladding; when the conducting light is reflected on the core-cladding interface, it will be scattered by these tiny crystals, and then the conducting light will be leaked. However, the attenuation coefficient changes little 
from in air to in liquid in a straight SEOF, and fiber bending must be used to increase the attenuation change.

In this paper, a liquid level sensor with a spiral bent SEOF is demonstrated. To investigate the sensor's response with different bent radius, a steadily changing spiral slope is applied to the sensor. The principle of the sensor is explained, while the design, simulation, and a prototype of the sensor are also presented. An InGaAlP Yellow LED (TLYH180P from TOSHIBA Inc.) with a typical peak wavelength of $590 \mathrm{~nm}$ is used as light source in all the experiments presented in this paper.

\section{Sensor Principle}

The basic schematic of a side-leaking OFLLS is shown in Figure 1. In this schematic, the height of the fiber is $H$, and the liquid level is $h$; the incident light power is $P_{i}$, while the emergent light power is $P_{R}$. In the side-leaking OFLLS, light propagating in fiber is attenuated exponentially to the submerged length with the coefficient related to the refractive index of the surrounding medium. In liquid level sensing, there are mainly two mediums: air and liquid, and the attenuation coefficient in liquid is larger than that in air. Define $\alpha_{a}$ and $\alpha_{l}$ as the attenuation coefficients in air and liquid, respectively, then the transmitted light attenuates with the coefficient $\alpha_{a}$ in the fiber exposed in air for a height of $(H-h)$, and the output power in this section is [16]

$$
P_{a}=P_{i} \exp \left[-\alpha_{a}(H-h)\right] .
$$

Then, the transmitted light attenuates with the coefficient $\alpha_{l}$ in the section of fiber in liquid for a height of $h$, and the output power in this section is

$$
P_{R}=P_{a} \exp \left(-\alpha_{l} h\right) .
$$

Replace $P_{a}$ in (2) with (1), and make a deformation to the exponential part of the formula; the output power can be simplified to be

$$
P_{R}=P_{i} \exp \left(-\alpha_{a} L\right) \exp \left[-\left(\alpha_{l}-\alpha_{a}\right) h\right] .
$$

In this equation, $\exp \left(-\alpha_{a} L\right)$ decides the ratio of receiving power to the incident power, and $\exp \left[-\left(\alpha_{l}-\alpha_{a}\right) h\right]$ decides the ratio of output change to the maximum output. If $\alpha_{l}$ is greater than $\alpha_{a}$ (this will be demonstrated later), the emergent power $P_{R}$ decreases as the level increases. If $\alpha_{l}-\alpha_{a}$ is small enough, $P_{R}$ responds linearly to liquid level change.

2.1. Light Attenuation of SEOF in Air and Liquids. In SEOF, the attenuation coefficient is larger in liquid than that in the air due to the different acceptance angle of the fiber. As shown in Figure 2, the propagating light is scattered by the tiny scattering crystals in the cladding. According to Rayleigh's equation [17], the scattered light intensity $I_{s}$ for an unpolarized source is given below:

$$
\begin{gathered}
I_{s}=A\left(1+\cos ^{2} \theta\right) I_{i}, \\
A=\frac{8 \pi^{4} N r^{6}}{\lambda^{4} R^{2}}\left(\frac{n_{1}^{2}-n_{0}^{2}}{n_{1}^{2}+2 n_{0}^{2}}\right)^{2},
\end{gathered}
$$

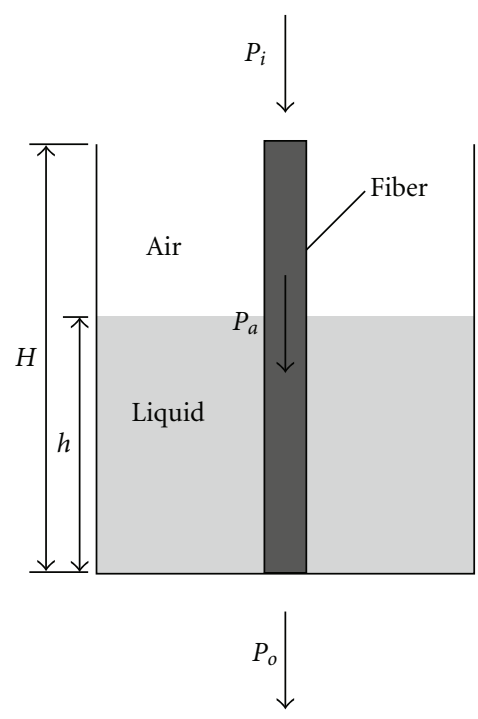

Figure 1: Schematic of a basic side-leaking OFLLS.

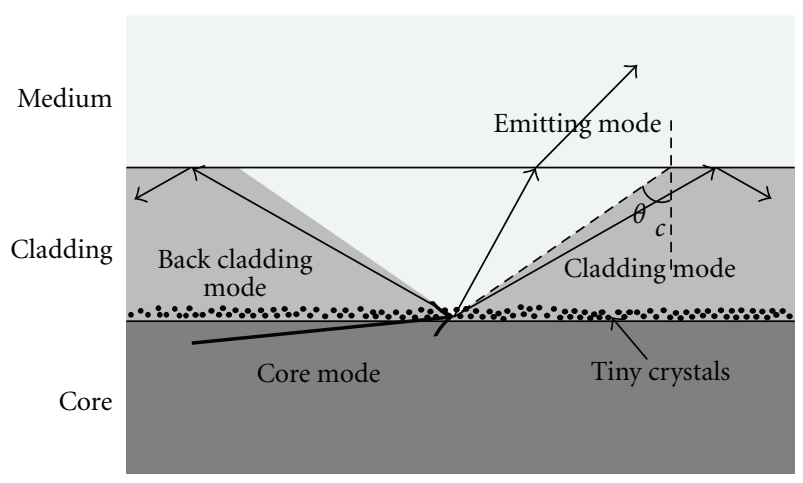

FIGURE 2: Light scattering in the cladding of SEOF.

where $I_{i}$ (Watts/meter squared) is the intensity of incident light, $\theta$ is the included angle between the scattered light and incident light, $A$ is a constant, in which $N$ is the number of scattering particles, $r$ is the radius of particle, $\lambda$ is the wavelength of incident light, $R$ is the distance between the particle and the observing point, $n_{1}$ is the index of particle, and $n_{0}$ is the index of the material around the particle. In the SEOF, the angle between transmitted light and fiber axis is very small, so it can be considered that $\theta$ approximately equals the angle between the scattering light and fiber axis.

The scattered light can be divided into three parts: part 1 is the backward cladding mode which transmits to the incident end; part 2 is the emitting mode which is refracted into the surrounding medium; part 3 is cladding mode which transmits along the fiber to the emergent end. Their boundaries are decided by the critical angle on the surface between the cladding and medium [18].

$$
\theta_{c}=\arcsin \left(\frac{n_{m}}{n_{\mathrm{cl}}}\right)
$$


where $n_{\mathrm{cl}}$ is the index of cladding and $n_{m}$ is the index of the medium outside of cladding. The power of cladding mode can be integrated as

$$
P_{r}=\int_{0}^{\arccos \left(n_{m} / n_{\mathrm{cl}}\right)} 2 \pi R^{2} I_{S}(\theta) \sin \theta d \theta,
$$

where $R$ is the same as the $R$ in (4), and it will be eliminated in the calculation. The total power of scattering light is

$$
P_{s}=\int_{0}^{\pi} 2 \pi R^{2} I_{S}(\theta) \sin \theta d \theta .
$$

Then, $P_{r}$ and $P_{s}$ can be calculated, and the ratio of $P_{r}$ to $P_{s}$ is

$$
\frac{P_{r}}{P_{s}}=\frac{1}{8}\left(4-\frac{3 n_{m} n_{\mathrm{cl}}^{2}+n_{m}^{3}}{n_{\mathrm{cl}}^{3}}\right) .
$$

The response of (8) to medium's index is shown in Figure 3, which indicates that the cladding mode power decreases while the index of the ambient medium of fiber increases. The value of (8) will reach the minimum value of 0 when $n_{m} \geq n_{\mathrm{cl}}$. In liquid level sensing, the index of the liquid is always larger than that of air, so there will be more power loss in fiber when the fiber is immersed in liquid than that in air. According to the definition of the fiber's attenuation coefficient [19], $\alpha_{l}$ is larger than $\alpha_{a}$.

2.2. Test of Attenuation Coefficients in Straight SEOF. The attenuation coefficients can be achieved by measuring the luminous exitance from the side of SEOF. Define $x$ as the distance from a point in the fiber from the incident end, and in a fragment of fiber at the location $x$ with a length of $\Delta x$ as it is shown in Figure 4, the incident light power is $P(x)$, the emergent light power is $P(x+\Delta x)$, and the power losses in this section can be calculated according to the definition of fiber's attenuation coefficient:

$$
P_{s}(x, \Delta x)=P_{i}[\exp (-\alpha x)-\exp (-\alpha x-\alpha \Delta x)] .
$$

Luminous exitance can be defined as the side-emitting power in per unit area, and it can be calculated as

$$
M(x)=\lim _{\Delta x \rightarrow 0} \frac{P_{s}(x, \Delta x)}{2 \pi r_{f} \Delta x},
$$

where $r_{f}$ is the radius of SEOF. Equation (10) can be simplified by series expansion, and the result is

$$
M(x)=\frac{\alpha P_{i}}{2 \pi r_{f}} \exp (-\alpha x)
$$

Since $\alpha$ is a very small value, (11) approximately equals

$$
M(x)=\frac{\alpha P_{i}}{2 \pi r_{f}} .
$$

Then, the attenuation coefficient, $\alpha$, is proportionate to the luminous exitance.

An experimental setup is established as it is shown in Figure 5 to test the fiber's attenuation coefficients in air and

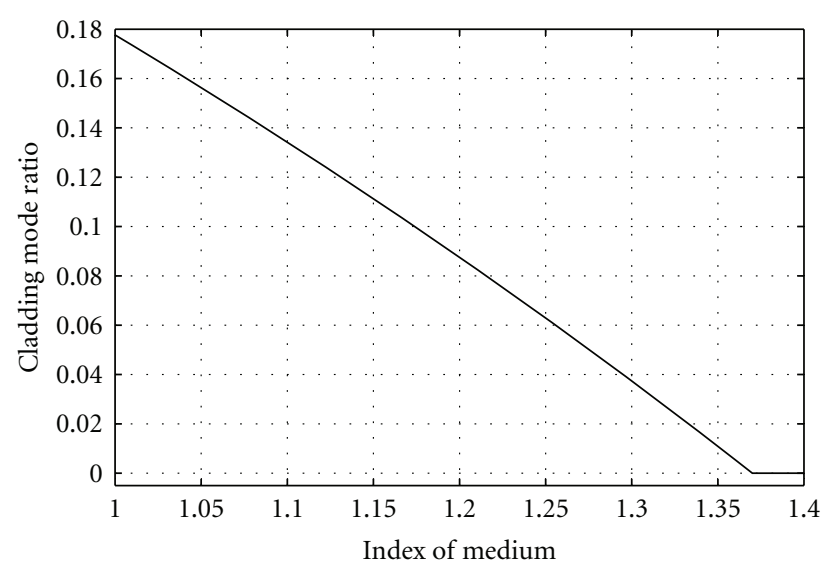

Figure 3: Cladding mode ratio versus medium index.

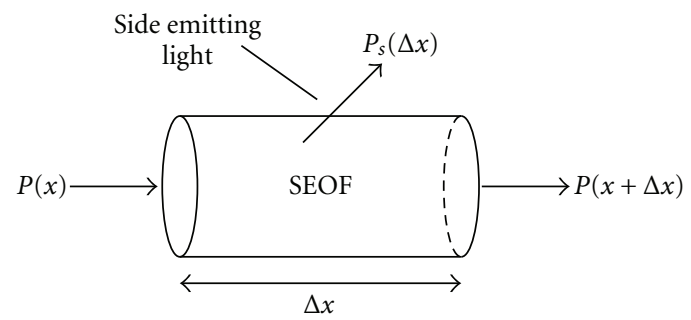

FIGURE 4: Side-emitting in a fragment of fiber.

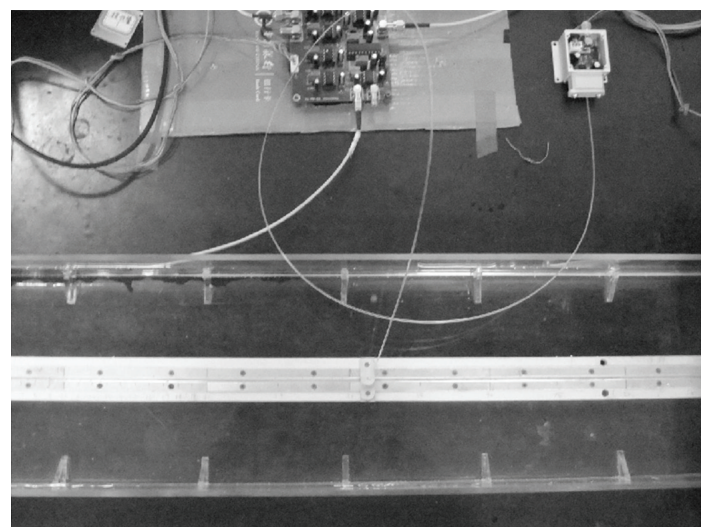

Figure 5: Experimental setup of attenuation coefficient measurement for straight SEOF.

in water. In this setup, an LED is used as the light source; a POF with $1 \mathrm{~mm}$ diameter is placed with its end facing the cladding of SEOF at location $x$, and it is used to guide the side-emitting light to the power meter. In the experiments, the $1 \mathrm{~mm}$ POF is moving from the input to the output end of SEOF, and its output power is recorded as $P_{p}(x)$ at location $x$. Then, the luminous exitance can be calculated as

$$
M(x)=\frac{P_{p}(x)}{\pi r_{p}^{2}},
$$


where $r_{p}$ is the radius of the $1 \mathrm{~mm}$ POF. Then, the attenuation coefficient can be calculated according to (12) and (13) as

$$
\alpha=\frac{2 r_{f} P_{p}(x)}{r_{p}^{2} P_{i}} .
$$

The measured result of the attenuation coefficients are $0.0045 / 0.0057(/ \mathrm{m})$ for air/water, respectively, in the middle of the fiber, and $\alpha_{l}-\alpha_{a}=0.0012(/ \mathrm{m})$. This is in accordance with the theoretical analysis.

\section{Enhancement of Attenuation Coefficient}

Because $\alpha_{l}$ is very close to $\alpha_{a}$, the output change is very small compared to the maximum output. In a 1-meter-long SEOF, the maximum change of output is only $0.12 \%$ compared to the maximum output. Such a small change of power is difficult to measure, because it will be overwhelmed by the temperature drift of the LED and photodiode. There are two methods to enhance the output change: increasing the difference between $\alpha_{l}$ and $\alpha_{a}$ and enlarging the submerged length of fiber, and both of them can be achieved by bending the SEOF as a spiral shape.

3.1. Attenuation Coefficient of Bent SEOF. It is demonstrated that the attenuation coefficient increases exponentially with the decreasing of bent radius [20]. In SEOF, there are too many modes of light, and the boundary conditions are very complex, so it is easier to get its attenuation coefficient by experiments than by calculation. In the experimental setup shown in Figure 6, the fiber is bent as a semicircular with radius varying from $1 \mathrm{~cm}$ to $8 \mathrm{~cm}$ by a step of $1 \mathrm{~cm}$. In this setup, light emitted by the LED is injected into the fiber directly, and the input power $P_{i}$ and output power $P_{o}$ are measured by a power meter. The experiments are taken with the fiber exposed in air (shown in Figure 6(a)) and submerged in water (shown in Figure 6(b)), respectively. Then, the attenuation coefficient per unit length can be calculated as

$$
\alpha_{L}=-\frac{\ln P_{o}-\ln P_{i}}{\pi R},
$$

where $R$ is the bent radius of the fiber. The SEOF's attenuation coefficient versus bent radius in the air is shown in Figure 7, and it indicates that the fiber's bend loss increases exponentially while the bent radius decreases. The difference between attenuation coefficients in air and in water versus bent radius is shown in Figure 8, and it shows the value $\left(\alpha_{l}-\alpha_{a}\right)$ increases when the bent radius decreases. With the tested value of $\alpha_{a}$ and $\alpha_{l}$, the sensor's response to liquid level can be simulated.

3.2. Light Attenuation in a Spiral SEOF. With a twodimensional bent fiber, the length of fiber immersed in liquid is not linear to the liquid level. Using a spiral shape can solve

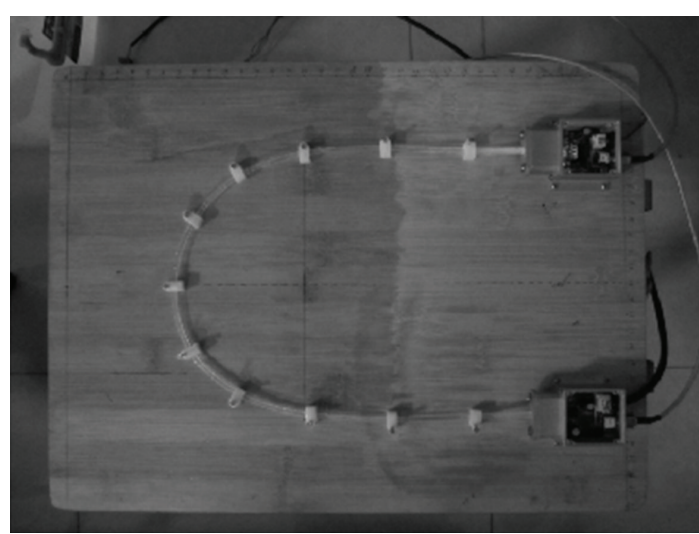

(a)

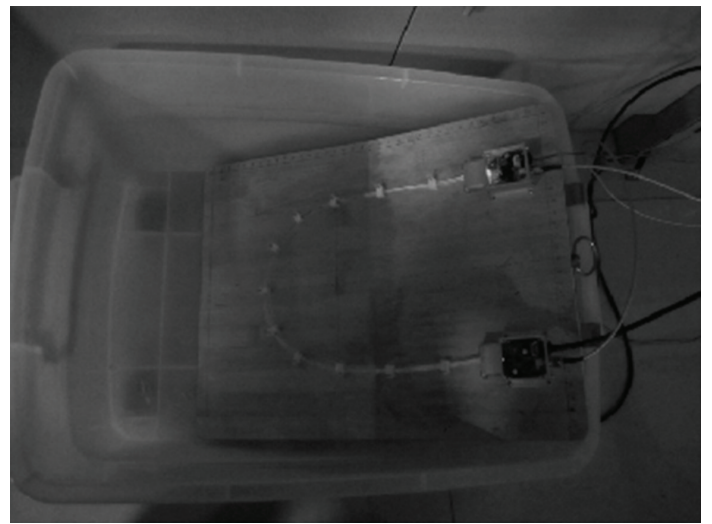

(b)

Figure 6: (a) and (b) are the experimental setup of attenuation coefficient measurement for bended SEOF in air and water.

this problem, and the immersed length can be enlarged in the same fiber. In a spiral line defined as

$$
\begin{gathered}
x=a \cos \theta, \\
y=a \sin \theta, \\
z=b \theta,
\end{gathered}
$$

$a$ is the radius of the core and $b$ is the slope of the spiral line. The arc length in the spiral line is

$$
S=\sqrt{z^{2}+(a \theta)^{2}} .
$$

Then, the ratio between arc length and height can be calculated by dividing $S$ by $z$, and the result will be

$$
K=\frac{\sqrt{a^{2}+b^{2}}}{b} .
$$

Define $\alpha_{H}$ as the power loss per unit height and $\alpha_{L}$ as the power loss per unit length, and $\alpha_{H}$ will be $K$ times as large as $\alpha_{L}$ according to the definition of the fiber's attenuation coefficient. This means $\alpha_{H}$ is always larger than $\alpha_{L}$. 


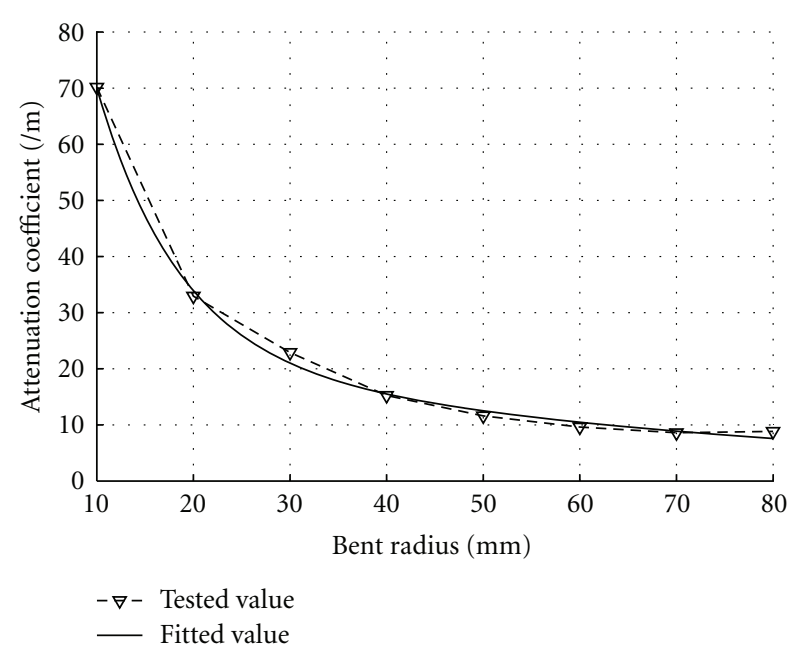

FIgURE 7: Attenuation coefficient of SEOF in air.

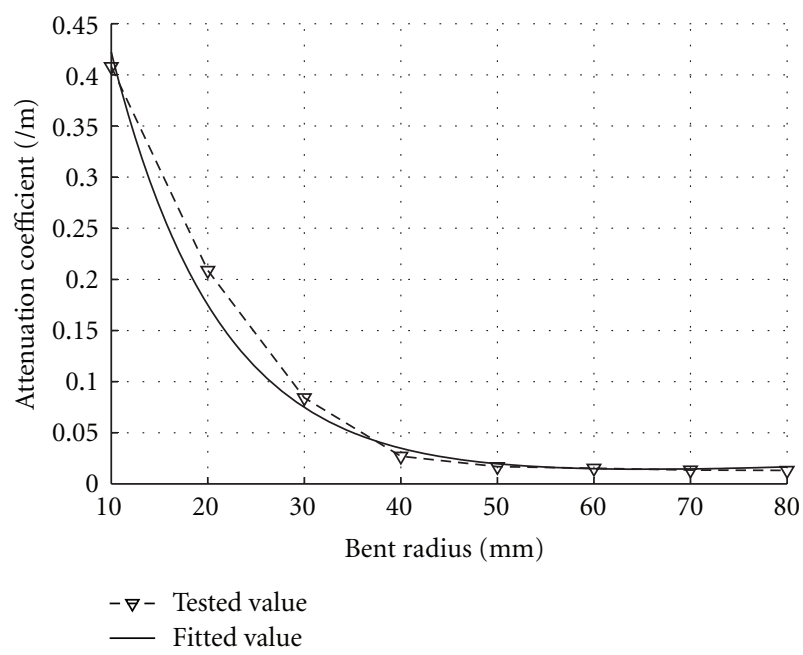

FIGURE 8: Difference between attenuation coefficient in air and liquid.

\section{Experimental Setup}

The schematic of the experimental system is shown in Figure 9. This system consists of a SEOF, two crabsticks, an LED, a photodiode, a signal processing circuit, and a data acquisition module DAM3056 to monitor the continuous level change. A PMMA SEOF of core/cladding diameter $2.6 / 3 \mathrm{~mm}$ and refractive indices $1.49 / 1.37$ (at $580 \mathrm{~nm}$ ) is used. The fiber is coiled downward on a crabstick as a spiral shape and turns upward along the other crabstick as it is shown in Figure 10. The track of fiber is fixed by the nails, and the function of the spiral is

$$
\begin{gathered}
x=12.5 \cos \theta, \\
y=12.5 \sin \theta, \\
z=-0.23 \theta^{2}+29.05 \theta .
\end{gathered}
$$

The spiral has a core radius of $12.5 \mathrm{~mm}$, a height of $900 \mathrm{~mm}$, a minimum bent radius of $15 \mathrm{~mm}$ at the top (shown

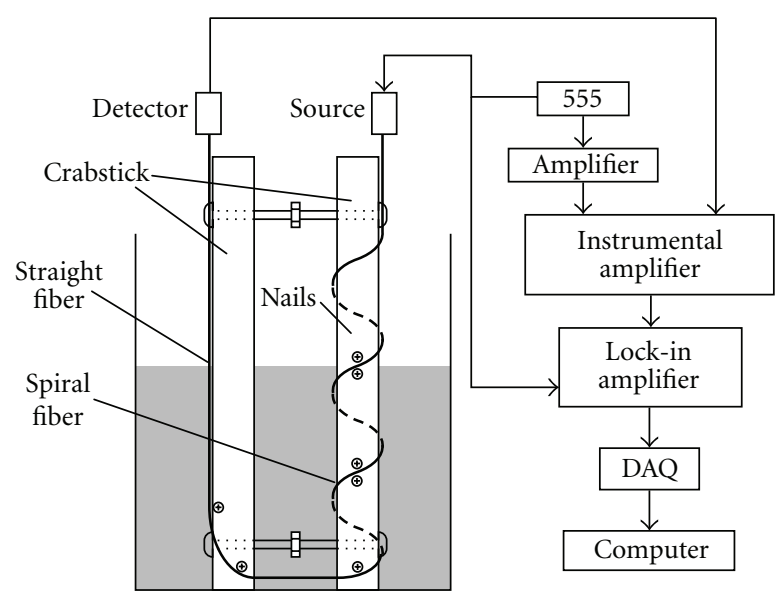

Figure 9: Schematic of liquid level sensor.

in Figure 10(b)), and a maximum bent radius of $80 \mathrm{~mm}$ at the bottom (shown in Figure 10(d)). The slope of the spiral is steadily decreased along the height; then, the sensor's response to liquid level with different bent radius can be tested. The simulated value of the sensor's response to level is shown in Figure 11. In this picture, the total variation of power is $7.9 \%$ of the maximum output, and this variation is 66 times as large as the output variation in a straight fiber; the sensitivity is stronger in the section with a smaller slope.

In the experimental system, the LED is driven by a $2.2 \mathrm{KHz}$ square wave generated by a 555 timer. The output power is detected by photodiode VTB8440 (PerkinElmer Optoelectronics, Inc.), of which the optical response extends from 330 to $720 \mathrm{~nm}$ and has peak sensitivity at $580 \mathrm{~nm}$. The output current of the photodiode is then transformed into voltage by a preamplifier, and this voltage is subtracted by a reference signal in an instrumentation amplifier, and then the limited change of power can be amplified. The reference signal is generated by amplifying the square wave to get a same amplitude and phase with the detector's signal at zero liquid level. The differential signal is then amplified and detected by a lock-in amplifier with a high signal-to-noise ratio, and the noise of the signal is reduced from $3 \mathrm{~V}$ to $0.02 \mathrm{~V}$. The output voltage of the lock-in amplifier is monitored by the data acquisition module and then sent to the computer.

\section{Results and Discussion}

Experiments are taken in water and aviation kerosene. In the water level experiment, a pump is used to let the level rise; after every $10 \mathrm{~mm}$ level changing, the flow controller is closed to hold the level and record the output voltage on the computer; when the level reaches $900 \mathrm{~mm}$, the pump is shut down, and the level will decrease under the pull of gravity, and the output voltage is also recorded after every $10 \mathrm{~mm}$ level changing. The experimental procedure is almost the same in the aviation kerosene level sensing, except using a hand pump to pump in the fuel.

The experimental results are shown in Figure 12. The solid and dashed curves are the experimental results in water, while the dash-dotted and dotted curves are the experimental 


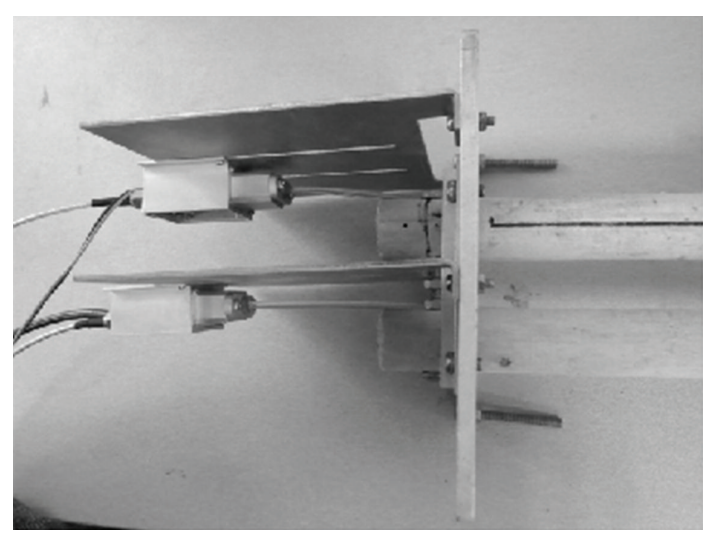

(a)

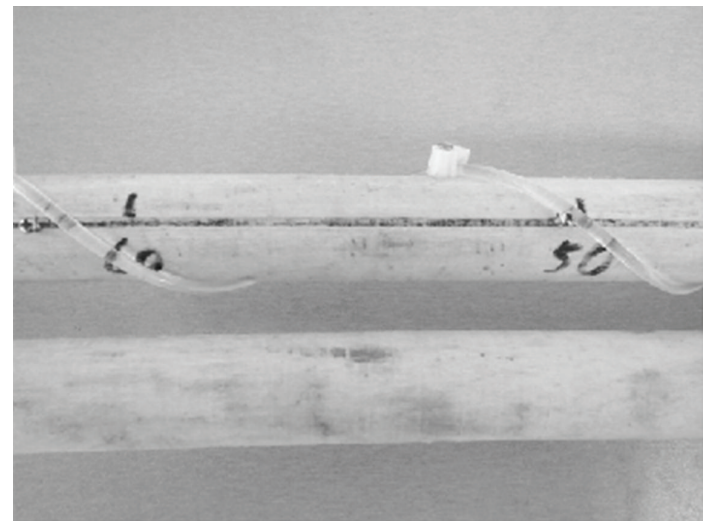

(c)

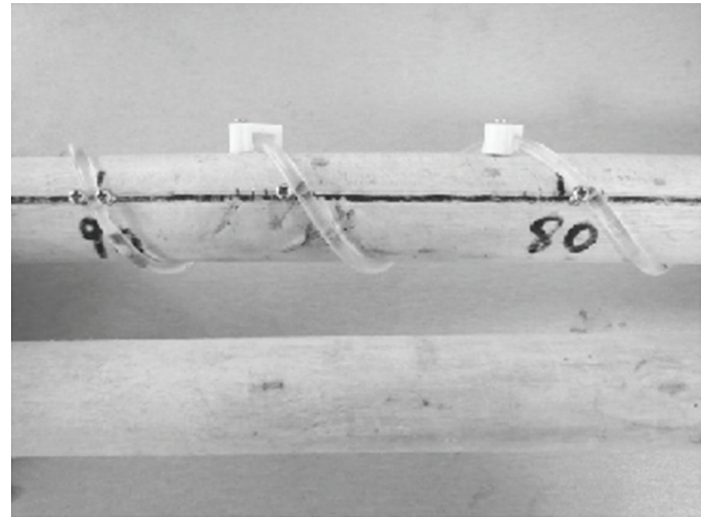

(b)

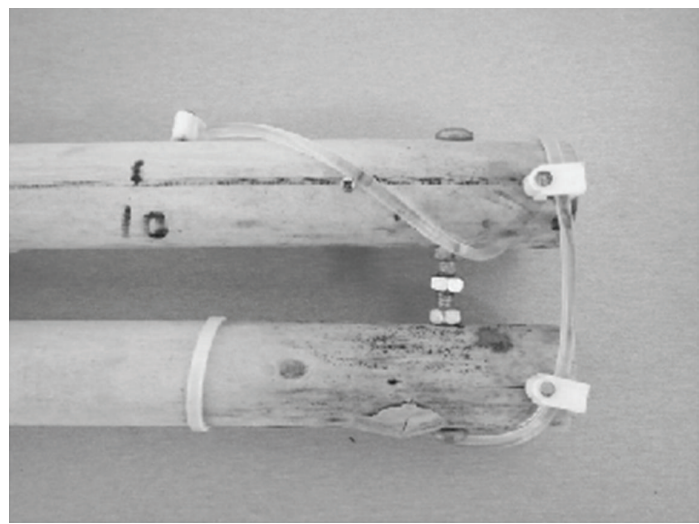

(d)

Figure 10: (a) source and detector; (b) top section of sensor; (c) middle section of sensor; (d) end section of sensor.

TABLE 1: Performance of several side-leaking optical fiber level sensors.

\begin{tabular}{lcc}
\hline Side-leaking method & Range $(\mathrm{m})$ & Resolution $(\mathrm{mm})$ \\
\hline Reduce cladding thickness & 1 & 62.5 \\
Remove zones of cladding & 1 & 25 \\
Fluorescent impurity-doped fiber & 1.5 & 40 \\
Fiber side-polish & 0.04 & 2.2 \\
Etched FBG & 0.02 & 2 \\
Tilted FBG & 0.02 & 4 \\
Side-emitting optical fiber & 0.9 & 10 \\
\hline
\end{tabular}

results in aviation kerosene. The sensor's output responses to the rising and decreasing of levels are shown in separate curves. In all the four curves, a sharp decrease of voltage happened in the beginning, this is caused by the turning section of fiber with a small radius at the bottom of the sensor. As it is shown in Figure 10(d), the fiber has a minimum radius of $12.5 \mathrm{~mm}$ in the turning section, which is much smaller than any other positions in the sensor. The average voltage drop is $3.2 \mathrm{~V}$ in aviation kerosene, about 1.28 times as large as the voltage drop in the water. The larger voltage change in aviation kerosene is caused by its larger refractive index of 1.43 , while the value is 1.33 in water. The drop of voltage at the beginning means the sensor is very sensitive to the existence of the liquid and can be used for low level detection. But for continuous liquid level sensing, this drop limits the amplification factor of the circuit. This disadvantage can be improved by covering the fiber's turning section with some opaque material to prevent the fiber from contacting with the liquid.

The curves are flatter in the following section. Coinciding with the simulated output in Figure 11, the sensor has a larger sensitivity in the section with a smaller bent radius. In the water level sensing the sensitivity of the sensor is $-1.14 \mathrm{mV} / \mathrm{mm}$ in the section of $(0 \sim 600)$ and $-3.65 \mathrm{mV} / \mathrm{mm}$ in the section of $(600 \sim 900)$. In the aviation kerosene level sensing, the sensitivity is $-1.31 \mathrm{mV} / \mathrm{mm}$ in the section of $(0 \sim 600)$ and $-3.73 \mathrm{mV} / \mathrm{mm}$ in the section of $(600 \sim 900)$. In the liquid level sensing, the measurement signal is easier 


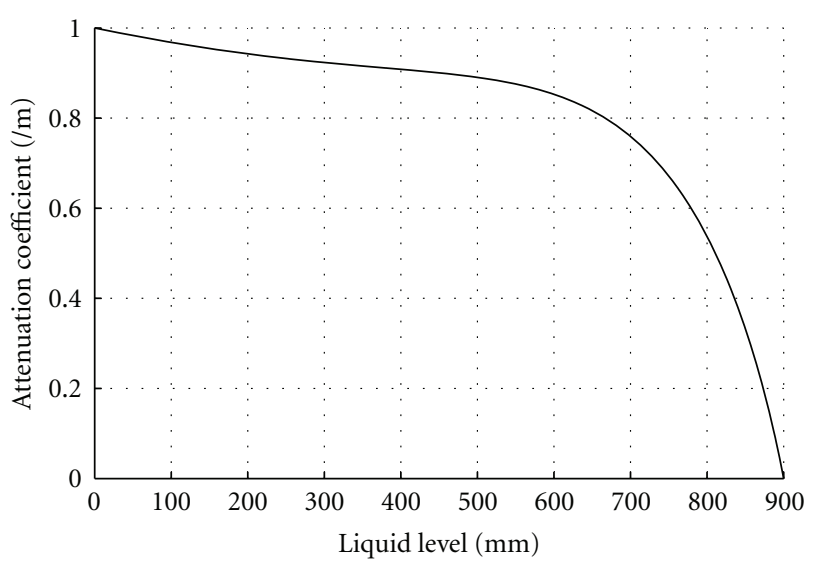

FIgURE 11: Simulation of sensor's output.

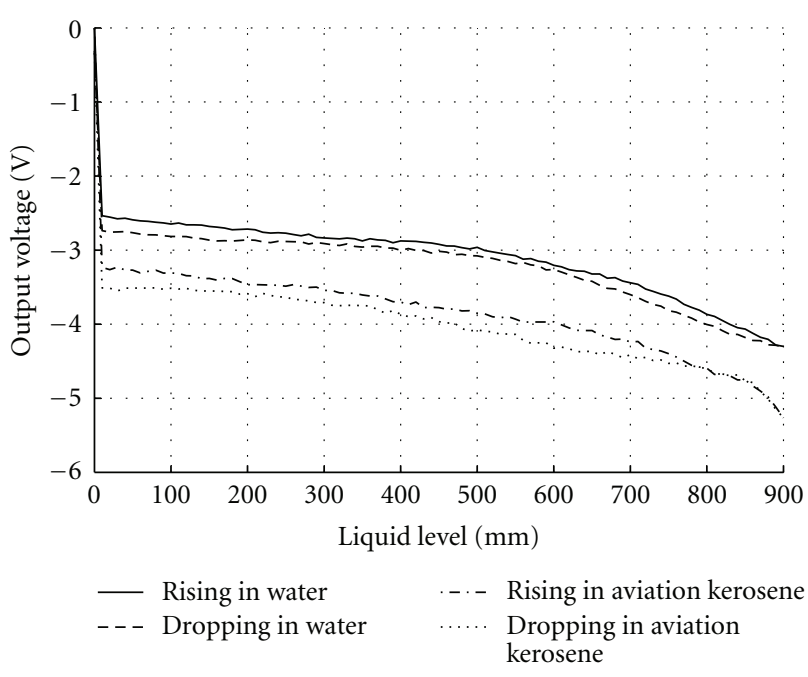

Figure 12: Output voltage versus liquid level.

to be amplified with a larger sensitivity. According to the experimental results, using a SEOF with a fixed bent radius of $15 \mathrm{~mm}$ will have a linear response to the liquid level, and the output power changing ratio will be enlarged.

The results show a hysteresis between the rising curve and the dropping curve. This hysteresis is caused by the liquid staying in the gap between fiber and crabstick during the level dropping. The difference between rising and dropping curves is larger in aviation kerosene than that in water, because the aviation kerosene has a larger viscosity than water (the viscosity of aviation kerosene/water is $1.25 / 1.005 \mathrm{~mm}^{2} / \mathrm{s}$ at $20^{\circ} \mathrm{C}$ ). The hysteresis of curves causes error in measurement, and half-embedding the fiber in a slot on the sensor's core will eliminate the gap between the fiber and the core; then the hysteresis will be limited.

The performances of this sensor and the OFLLSs mentioned in the introduction are compared in Table 1. In this table, the sensors with a large measurement range have a limited resolution, while the sensors with a high resolution have a limited range. In aircraft fuel measurement, the required measurement range is $1 \mathrm{~m}$, and the required resolution is $5 \mathrm{~mm}$. Among the sensors showed in Table 1, the sensor described in this paper is the one which mostly meets the requirement in aircraft fuel measurement.

\section{Conclusions}

In this paper, a novel optical fiber level sensor was proposed, based on a spiral bent side-emitting optical fiber, which is produced by cladding material crystallization. The principle of the sensor was interpreted by analyzing the light scattering in the SEOF, and experimental results were presented to explain the reason for using a spiral bent SEOF. A prototype with a steadily changing spiral slope was designed to demonstrate the sensor principle and test the sensor's response with different fiber bent radius. The experimental results taken in water and aviation kerosene showed coincidence to the simulated results. There are also several disadvantages in this sensor, like the long-time instability, the sharp output decrease near the zero level, the hysteresis during level rising and dropping, and the nonlinear response to liquid level. By solving these problems, the sensor's performance can be improved and be suitable for the fuel level measurement in vehicles like a plane, truck, or ship and the level sensing in liquid with low viscosity.

\section{Authors' Contribution}

C. Zhao elaborated the basic principle of the sensor, proposed a new method to increase the sensor's sensitivity by fiber bending, realized this sensor, and made experiments with this sensor. L. Ye clarified the theory of side-leaking fiber level sensor and proposed the idea of using a side-emitting optical fiber in this sensor. J. Ge explained the reason of fiber's bend loss and designed experiments to test the fiber's bend loss. X. Yu made experiments to test the fiber's bend loss and took part in the experiments of the sensor.

\section{Acknowledgments}

This work is partly supported by the National Nature Science Foundation of China (61104202), and the authors wish to thank Ms. Cheng Yi for the excellent work during this research.

\section{References}

[1] R. Langton, C. Clark, M. Hewitt, and L. Richards, Aircraft Fuel Systems. Chichester, John Wiley \& Sons, United Kingdom, 2009.

[2] J. D. Weiss, "Fluorescent optical liquid-level sensor," Optical Engineering, vol. 39, no. 8, pp. 2198-2213, 2000.

[3] K. R. Sohn and J. H. Shim, "Liquid-level monitoring sensor systems using fiber Bragg grating embedded in cantilever," Sensors and Actuators A, vol. 152, no. 2, pp. 248-251, 2009.

[4] H. J. Sheng, M. Y. Fu, T. C. Chen, W. F. Liu, and S. S. Bor, "A lateral pressure sensor using a fiber Bragg grating," IEEE Photonics Technology Letters, vol. 16, no. 4, pp. 1146-1148, 2004.

[5] H. K. Singh, S. K. Chakroborty, H. Talukdar, N. M. Singh, and T. Bezboruah, "A New Non-Intrusive optical technique to measure transparent liquid level and volume," IEEE Sensors Journal, vol. 11, no. 2, pp. 391-398, 2011. 
[6] A. A. Kazemi, C. Yang, and S. Chen, "Fiber optic cryogenic liquid level detection system for space applications," in Photonics in the Transportation Industry: Auto to Aerospace II, A. A. Kazemi, Ed., p. 73140A, 2009.

[7] F. Pérez-Ocón, M. Rubiño, J. M. Abril, P. Casanova, and J. A. Martínez, "Fiber-optic liquid-level continuous gauge," Sensors and Actuators A, vol. 125, no. 2, pp. 124-132, 2006.

[8] G. Betta, L. Ippolito, A. Pietrosanto, and A. Scaglione, "Optical fiber-based technique for continuous-level sensing," IEEE Transactions on Instrumentation and Measurement, vol. 44, no. 3, pp. 686-689, 1995.

[9] G. Betta, A. Pietrosanto, and A. Scaglione, "A digital liquid level transducer based on optical fiber," IEEE Transactions on Instrumentation and Measurement, vol. 45, no. 2, pp. 551-555, 1996.

[10] J. D. Weiss, "A novel fiber-optic fluid interface sensor. Sensors," 2008, http://www.sensorsmag.com/da-control/a-novel-fiberoptic-fluid-interface-sensor-1476.

[11] M. Lomer, A. Quintela, M. López-Amo, J. Zubia, and J. M. López-Higuera, "A quasi-distributed level sensor based on a bent side-polished plastic optical fibre cable," Measurement Science and Technology, vol. 18, no. 7, article no. 061, pp. 22612267, 2007.

[12] B. Yun, N. Chen, and Y. Cui, "Highly sensitive liquid-level sensor based on etched fiber bragg grating," IEEE Photonics Technology Letters, vol. 19, no. 21, pp. 1747-1749, 2007.

[13] Q. Jiang, D. Hu, and M. Yang, "Simultaneous measurement of liquid level and surrounding refractive index using tilted fiber Bragg grating," Sensors and Actuators A, vol. 170, no. 1-2, pp. 62-65, 2011.

[14] J. Spigulis, D. Pfafrods, M. Stafeckis, and W. Jelinska-Platace, "Glowing optical fiber designs and parameters," in Optical Inorganic Dielectric Materials and Devices, A. Krumins, D. K. Millers, A. R. Sternberg, and J. Spigulis, Eds., Proceedings of SPIE, p. 231, Riga, Latvia, 1997.

[15] S. Katsuhiko, T. Tsuneo, T. Yasuteru, and I. Kikue, "Illuminating plastic optical fiber and its manufacture," Japan Patent 06118236, 1992.

[16] J. Hecht and L. Long, Understanding Fiber Optics, Publishing House of Electronics Industry, Beijing, China, 5th edition, 2003.

[17] J. C. Stover, Optical Scattering: Measurement and Analysis, SPIE-International Society for Optical Engineering, Bellingham, Wash, USA, 2nd edition, 1995.

[18] M. Born, E. Wolf, and A. B. Bhatia, Principles of Optics: Electromagnetic Theory of Propagation, Interference and Diffraction of Light, Cambridge University Press, 7th edition, 1999.

[19] J. Hecht and L. Long, Understanding Fiber Optics, Prentice Hall Columbus, 4th edition, 2002.

[20] D. Marcuse, "Curvature loss formula for optical fibers," The Journal of the Optical Society of America, vol. 66, no. 3, pp. 216220, 1976. 

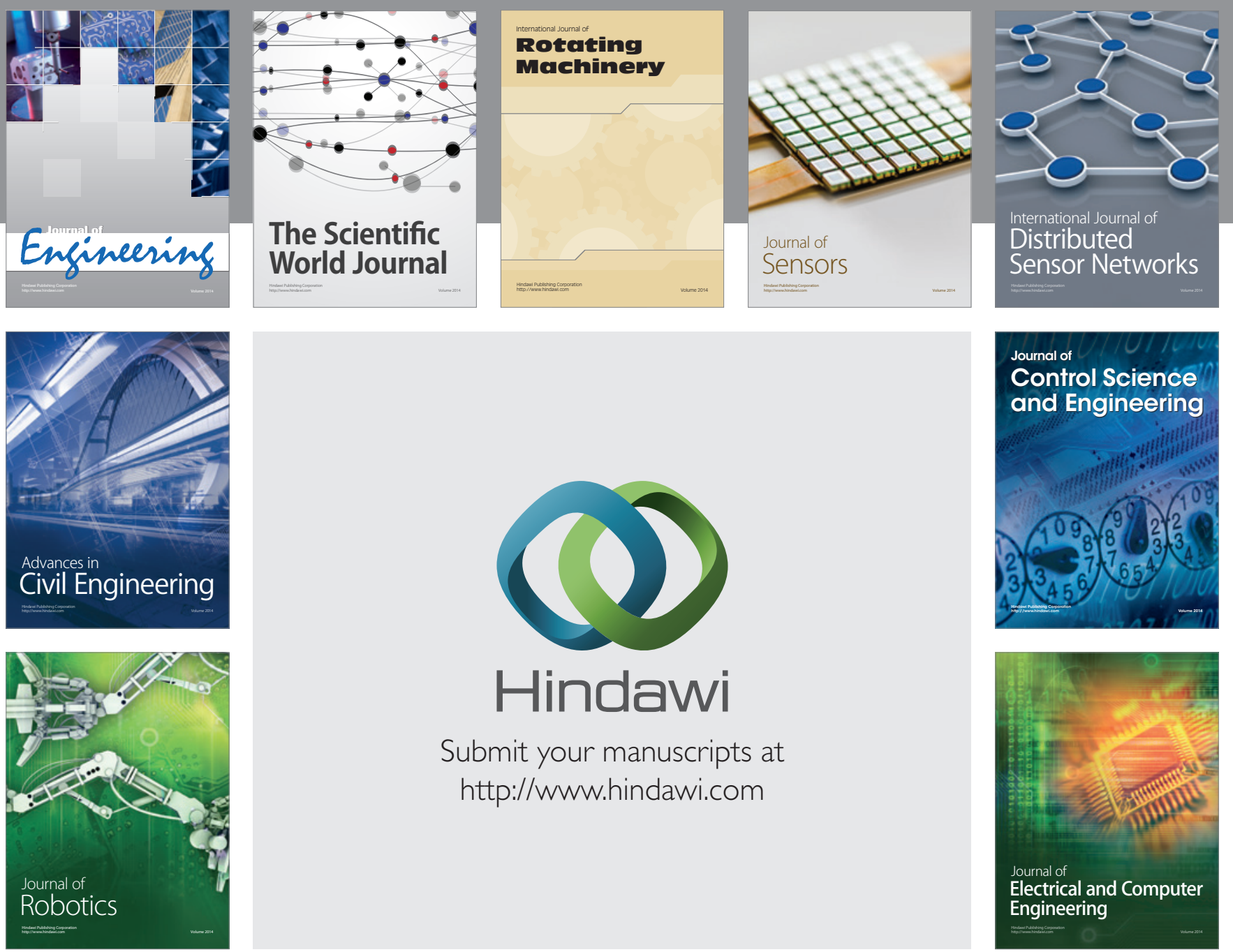

Submit your manuscripts at

http://www.hindawi.com
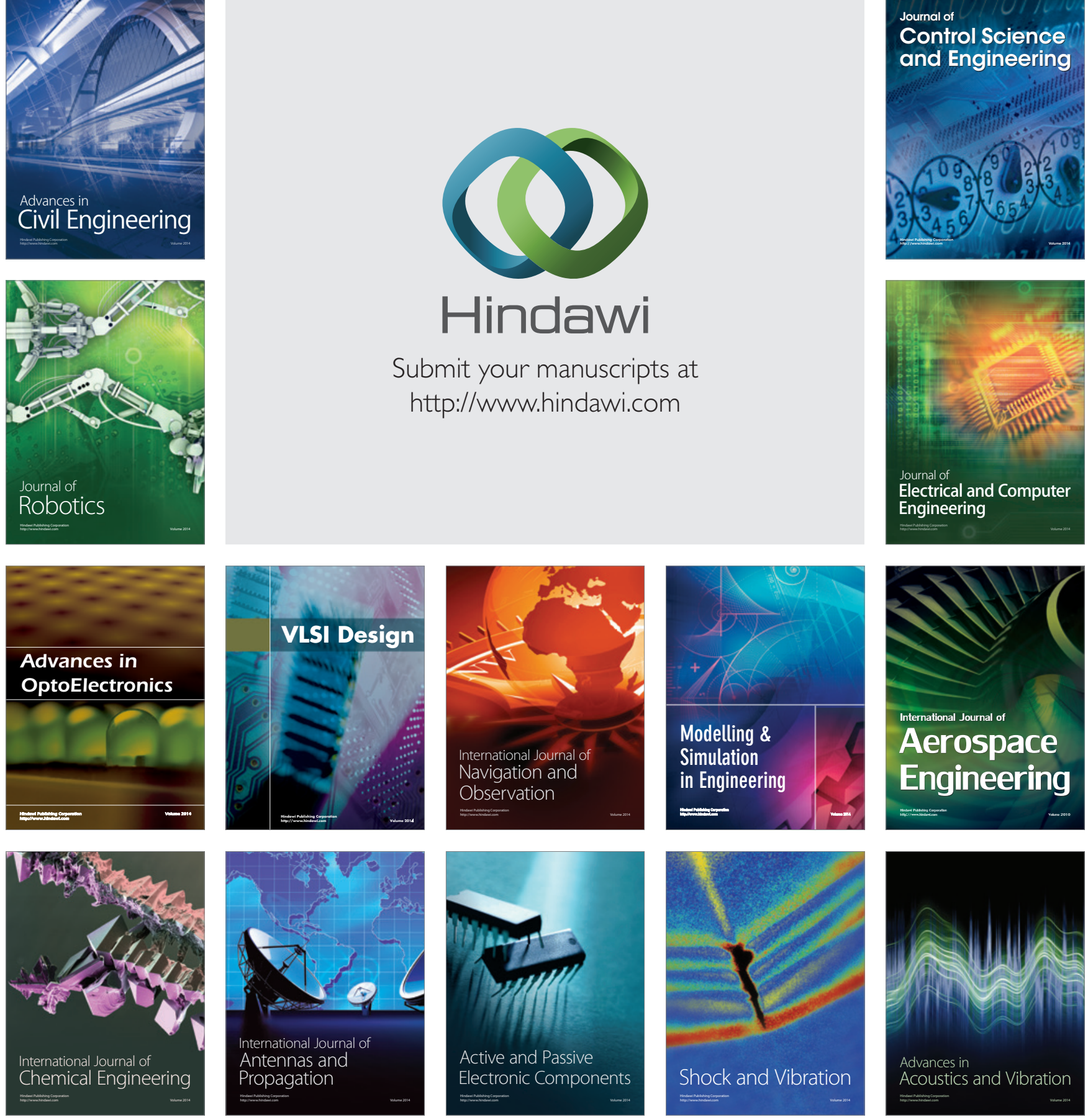